The Journal of Laryngology \& Otology

http://journals.cambridge.org/JLO

Additional services for The Journal of Laryngology \& Otology:

Email alerts: $\underline{\text { Click here }}$

Subscriptions: $\underline{\text { Click here }}$

Commercial reprints: $\underline{\text { Click here }}$

Terms of use : $\underline{\text { Click here }}$

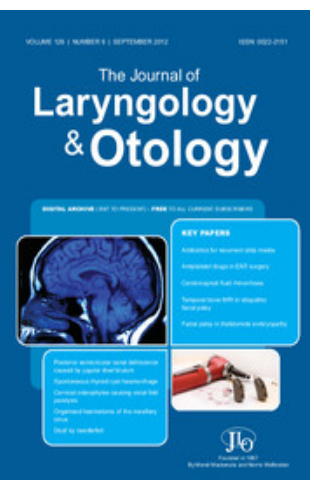

\title{
Guillotine versus dissection tonsillectomy: randomised, controlled trial
}

S J Frampton, M J A Ward, V S Sunkaraneni, H Ismail-Koch, Z A Sheppard, R J Salib and P K Jain

The Journal of Laryngology \& Otology / FirstView Article / September 2012, pp 1 - 8

DOI: 10.1017/S002221511200196X, Published online:

Link to this article: http://journals.cambridge.org/abstract_S002221511200196X

How to cite this article:

S J Frampton, M J A Ward, V S Sunkaraneni, H Ismail-Koch, Z A Sheppard, R J Salib and P K Jain Guillotine versus dissection tonsillectomy: randomised, controlled trial. The Journal of Laryngology \& Otology, Available on CJO doi:10.1017/ S002221511200196X

Request Permissions : $\underline{\text { Click here }}$ 


\title{
Guillotine versus dissection tonsillectomy: randomised, controlled trial
}

\author{
S J FRAMPTON ${ }^{1} \&$ M J A WARD ${ }^{1}$, V S SUNKARANENI ${ }^{2}$, H ISMAIL-KOCH ${ }^{2}$, \\ Z A SHEPPARD ${ }^{3}$, R J SALIB ${ }^{2}$, P K JAIN $^{1}$ \\ ${ }^{1}$ Department of Otolaryngology, Poole Hospital NHS Foundation Trust, ${ }^{2}$ Department of Otolaryngology, \\ University Hospital Southampton NHS Foundation Trust, and ${ }^{3}$ School of Health and Social Care, Bournemouth \\ University, $U K$
}

\begin{abstract}
Objective: This trial aimed to compare the guillotine technique of tonsillectomy with 'cold steel' dissection, the current 'gold standard'.

Design: A single centre, randomised, controlled trial.

Methods: One hundred children aged 3 to 11 years who were listed for bilateral tonsillectomy were recruited. Patients had one tonsil removed by each technique, and were blinded to the side. The operative time, intraoperative blood loss, haemostasis requirement and post-operative pain scores were recorded and compared.

Results: Operative time and intra-operative blood loss were both significantly less for the guillotine technique $(p<0.001)$ and there was a significantly reduced haemostasis requirement $(p<0.001)$. Pain was also less on the guillotine side $(p<0.001)$. There were no tonsillar remnants or palatal trauma for either technique. There was no significant difference between techniques in the frequency of secondary haemorrhage.

Conclusion: This study provides level Ib evidence that guillotine tonsillectomy in children with mobile tonsils is an effective and time-efficient procedure which produces less intra-operative blood loss and post-operative pain than cold steel dissection.
\end{abstract}

Key words: Tonsillectomy; Pain, Postoperative; Postoperative Complications; Efficiency

\section{Introduction}

Removal of tonsillar tissue was first described by Cornelius Caesus in $30 \mathrm{AD}$, and has since become one of the most common procedures performed by otolaryngologists. ${ }^{1}$ Over 50000 tonsillectomies were performed in National Health Service trusts in England between July 2003 and September 2004. ${ }^{2}$ Various tonsillectomy instruments and techniques have evolved over time, with the first tonsillar guillotine being developed in the eighteenth century. ${ }^{1,3}$ Perhaps the most recognised is Popper's haemostatic guillotine, developed and introduced in 1929 by Otto Oswald Popper. Its two-blade design was revolutionary, with the crushing blade sealing vessels before the deployment of the cutting blade (Figure 1). ${ }^{1}$

In the early twentieth century, guillotine tonsillectomy (using ether anaesthesia and no endotracheal tube) gained popularity as a quick and relatively bloodless technique. Immediately following the procedure, patients were placed on their side and left in the recovery position until any bleeding had ceased. As the twentieth century progressed, advances in anaesthesia, including endotracheal intubation, facilitated a less time-pressured procedure, and the use of the tonsil guillotine diminished. It is unclear whether this was due to concerns regarding bleeding and tonsillar remnants, or to the advent of newer, more fashionable techniques and instruments. ${ }^{1}$ By the end of the $1990 \mathrm{~s}$, only 12.5 per cent of respondents to a survey of British otolaryngologists reported regularly performing guillotine tonsillectomy. ${ }^{3}$

The 'cold steel' dissection tonsillectomy technique involves removal of the tonsils using scissors and a blunt tonsil dissector. It is currently the most commonly performed technique, being used in 53 per cent of tonsillectomy cases in a recent National Prospective Tonsillectomy Audit. ${ }^{2}$ Other technological advances include the use of monopolar or bipolar diathermy, carbon dioxide and potassium titanyl phosphate lasers, coblation, and the harmonic scalpel; 


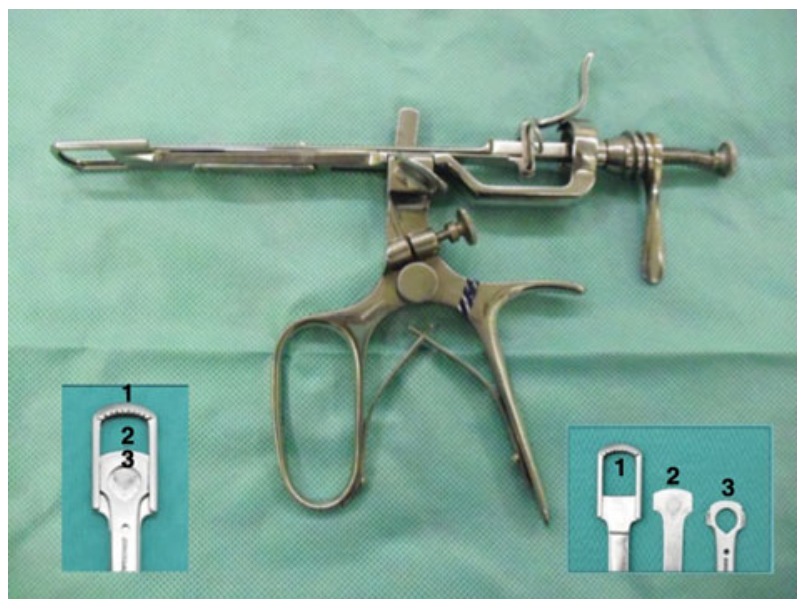

FIG. 1

Popper's haemostatic guillotine. Insets show: (1) fenestra; (2) crushing (haemostatic) blade; and (3) cutting blade.

however, all have their drawbacks. In the above-mentioned national audit, tonsillectomy using coblation or diathermy instruments for dissection was shown to result in higher rates of post-operative haemorrhage than cold steel techniques. ${ }^{2}$

There have been no well controlled trials justifying concerns about guillotine-related haemorrhage, incomplete tonsillar excision or inadvertent trauma to local structures. However, a recent retrospective analysis of 168 guillotine tonsillectomies suggested that intraoperative bleeding was minimal and that complication rates were equivalent to those of dissection tonsillectomy. ${ }^{4}$

The current study aimed to prospectively compare guillotine and dissection tonsillectomy with respect to operative time, intra-operative bleeding, post-operative pain and secondary haemorrhage.

\section{Materials and methods}

\section{Patient selection and randomisation}

This study was approved by the Dorset local research ethics committee (approval number 06/Q2201/49).

The sample size calculation assumed 90 per cent power to detect a preference rate of 70 per cent for one tonsillectomy technique being less painful, the morning after surgery, compared to 50 per cent that would be expected by chance. This calculation specified a sample size of 62 individuals; however, 100 children were recruited to allow for a 25 per cent prevalence of failure to express a preference, and a 20 per cent withdrawal rate.

Exclusion criteria included concurrent adenoidectomy, history of peritonsillar abscess, and any personal or family history of bleeding diathesis.

If a tonsil intended for guillotine excision had been found intra-operatively to be too fibrotic to mobilise, or too large to fit through the guillotine, then the tonsil would have been removed by the dissection technique and the event noted. However, this situation did not arise.
We recruited 100 children between the ages of 3 and 12 years (mean age, 7.05 years; standard deviation (SD), 2.48 years) scheduled for tonsillectomy for recurrent tonsillitis between January 2007 and August 2009. Written and verbal consent was obtained from parents and, where feasible, from the child themselves. Each patient recruited into the trial had one tonsil removed with the guillotine and the other by cold steel dissection, thereby acting as their own control.

The side for guillotine tonsillectomy was randomised on the day of surgery. Fifty blank, sealed envelopes containing the words 'left guillotine' and 50 the words 'right guillotine' were mixed together and kept at the operating theatre reception desk. An envelope was randomly selected for each patient by a member of the nursing staff. Only the operating room staff knew the outcome of the selection. All tonsillectomies were performed by the same surgeon (PKJ).

\section{Surgical technique}

Guillotine technique. Equipment used for guillotine tonsillectomy is shown in Figure 2. Patients were placed in the Rose position under general anaesthesia with an endotracheal tube. A Doyen mouth gag was engaged on one side of the mouth. This ensured that the tonsil and surrounding tissue remained relaxed and so could be passed through the fenestra of the guillotine. Right-sided tonsils were removed by holding the guillotine in the left hand and approaching from the left side of the mouth, and vice versa. The lower pole and posterior border of the tonsil were first engaged in the fenestra to draw the tonsil forward. The whole tonsil was then passed through the fenestra with the thumb of the surgeon's free hand placing pressure over the palatoglossal fold to facilitate tonsil medialisation. Ensuring that the whole tonsil had passed through the guillotine fenestra, the crushing blade was deployed firmly through the loose areolar tissue between the tonsil and the superior constrictor muscle. Pressure was maintained for 2 minutes by tightening the screw attached to the crushing blade. After 2 minutes, the separate cutting blade was deployed and the tonsil was removed. Following the excision, the anterior and posterior pillars were often folded and temporarily adherent to each another. Replacement of the Doyen gag at this stage with the Boyle-Davis gag and the Jain ' $A$ ' frame enabled better visualisation of the tonsillar fossa as the mucosal folds separated..$^{5}$ The fossa was inspected for tonsillar remnants and potential bleeding points using the Jain insulated pillar retractor. Haemostasis was performed using tonsil swabs to absorb any blood, and using bipolar diathermy at a setting of 8 Watts.

Dissection technique. Dissection tonsillectomy was not commenced until haemostasis had been adequately secured on the guillotine side. The dissection technique was performed in a standard manner, having initially placed a Boyle-Davis mouth gag. Scissors were used 


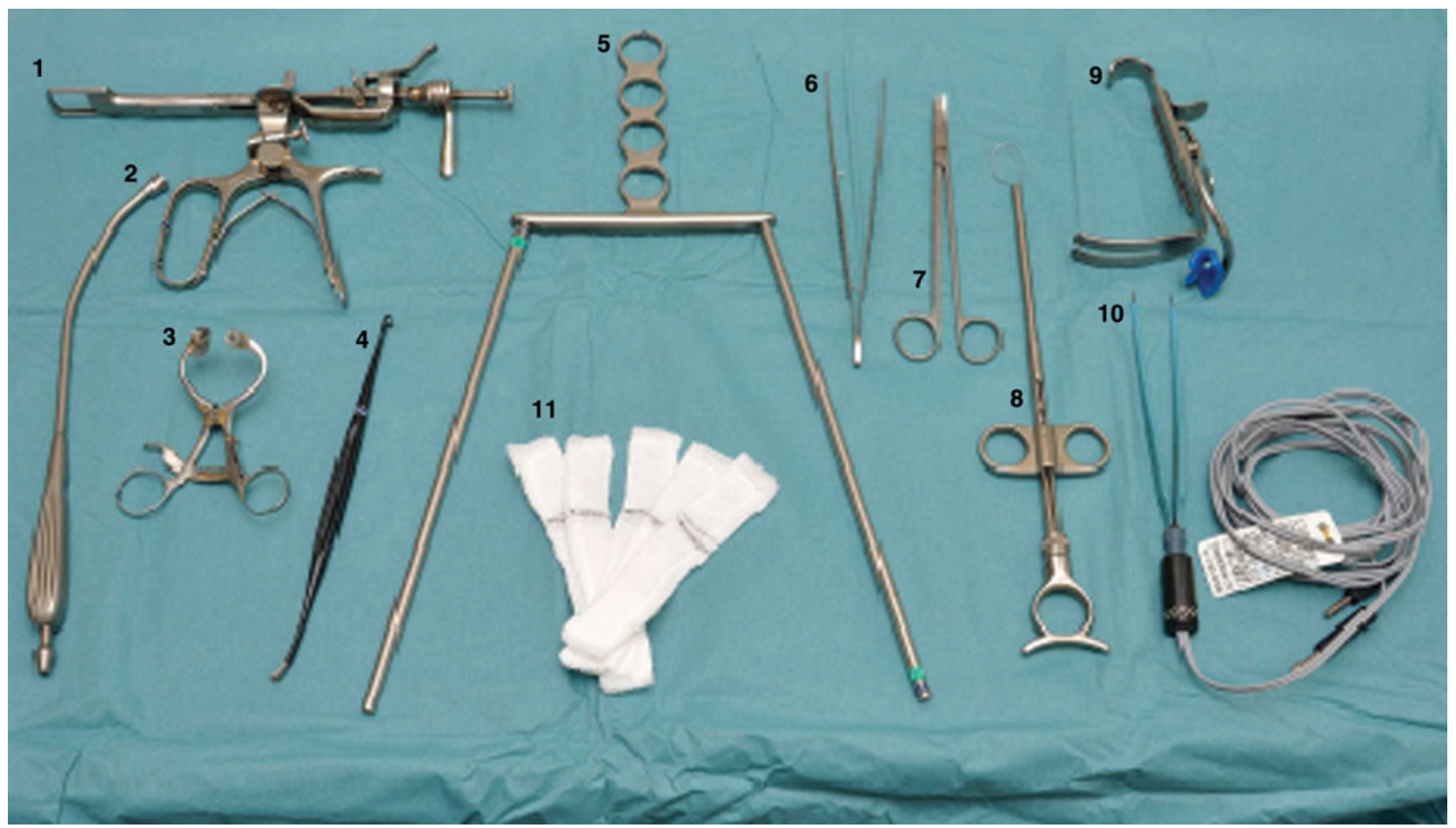

FIG. 2

Equipment for both dissection and guillotine procedures: (1) Popper's haemostatic guillotine; (2) Yankauer sucker; (3) Doyen mouth gag; (4) Jain insulated tonsil dissector and pillar retractor; (5) Jain tonsil 'A' frame; ${ }^{5}$ (6) Waugh forceps; (7) McIndoe scissors; (8) tonsil snare; (9) Boyle-Davis mouth gag; (10) bipolar diathermy forceps; and (11) tonsil swabs.

to make the initial incision in the palatoglossal fold, and an insulated Jain dissector was used to dissect the tonsil while tension was applied by pulling the tonsil medially with Dennis-Brown forceps. A tonsil snare was used to excise the inferior pole. Tonsil swabs and $8 \mathrm{~W}$ bipolar diathermy were used for haemostasis if required.

\section{Operative time}

On the guillotine side, a stopwatch was started when the Doyen mouth gag was inserted. The stopwatch was stopped, and the time recorded to the nearest minute, when the Boyle-Davis mouth gag was removed following achievement of haemostasis.

On the dissection side, the time was recorded from insertion to removal of the Boyle-Davis gag.

\section{Intra-operative bleeding}

Blood loss was measured by comparing the weight of blood-soaked tonsil swabs with that of unused, dry swabs, with a $1 \mathrm{~g}$ difference estimated to be equivalent to $1 \mathrm{ml}$ of blood. The quantity of any suctioned blood was measured by calculating the weight gain of the suction apparatus. Blood loss was recorded to the nearest $\mathrm{ml}$. The use of techniques to stop bleeding (i.e. diathermy, ties etc) was also recorded.

\section{Post-operative pain}

Post-operative pain on each side was recorded using a six-point visual pain score. Children, supervised by their parents, were asked to score their pain on each side at five post-operative time points. These were: immediately on waking in the recovery room, at 2 and 5 hours post-operatively on the ward, on post-operative day one, and finally on post-operative day seven by means of a telephone questionnaire. Families were given a copy of the scoring sheet to take home with them, to act as an aide memoire. Pain scores were recorded by a member of the research team. All patients received our standard departmental post-operative analgesia.

\section{Data analysis}

Data were analysed using the Statistical Package for the Social Sciences for Windows version 16.0 and the Predictive Analytics Software version 18 software programs.

Means and SDs were calculated as measures of location and spread for the normally distributed variables, and as medians and inter-quartile ranges for the non-normally distributed variables. For consistency of presentation of medians and inter-quartile ranges for the non-normally distributed data, the more conservative Wilcoxon signed rank test was used to analyse differences in operative time and intra-operative blood loss; however, findings were consistent with analysis using paired $t$-tests.

Area under the curve analysis was used to summarise pain scores over time, and the Wilcoxon signed rank test was used to compare the area under the curve for 
the two techniques. Because of the paired nature of the data, McNemar's test was used to compare the proportion of tonsils requiring either no haemostasis, or swabs and/or diathermy, for each technique. McNemar's test was also used to compare the proportion of tonsils with secondary haemorrhage, for each technique.

\section{Results and analysis}

Of the 100 children recruited, 55 were girls and 45 boys. Guillotine tonsillectomy was performed successfully in all cases, and removed 50 left and 50 right tonsils. Tonsillectomy was performed as a day case in 94 cases. Of the remaining six patients, five lived outside of the approved travelling distance for day surgery cases, and one was observed overnight following laryngospasm on extubation.

\section{Operative time and intra-operative bleeding}

The operative time for the guillotine technique (median: 6 minutes; inter-quartile range (IQR): 5-7 minutes) was less than that for the dissection technique (median: 10.5 minutes; IQR: 9-13 minutes), and this difference was statistically significant (Wilcoxan twosided $p<0.001$ ), (Table I, Figures 3 and 4).

Intra-operative blood loss was also significantly less for guillotine tonsillectomy (median: $1 \mathrm{ml}$; IQR: 0-2 $\mathrm{ml}$ ) compared to the dissection technique (median: $17 \mathrm{ml}$; IQR: $10-25 \mathrm{ml} ; p<0.001$ ) (Table I, Figures 5 and 6 ). It was noted that the use of suction in the tonsillar fossae seemed to promote bleeding following both techniques; therefore, after the first two cases suction was no longer used. Even after discounting these two cases, the median intra-operative blood loss was still significantly less for guillotine tonsillectomy (median $1 \mathrm{ml}$; inter-quartile range, $0-2 \mathrm{ml}$ ) than for the dissection technique (median $16.5 \mathrm{ml}$; interquartile range, $10-24 \mathrm{ml} ; p<0.001)$. Guillotine tonsillectomy also required less extensive haemostasis, with
37 per cent of cases requiring no haemostasis and the remaining 63 per cent requiring swabs and bipolar diathermy. In all cases of dissection tonsillectomy, swabs and bipolar diathermy were required $(p<0.001$, McNemar two-sided test). No ties were used in either group for haemostasis.

\section{Post-operative pain}

The overall pain scores reported by patients in the week after their operation were less for the guillotine side (area under the curve analysis, $n=100$; median, 172; inter-quartile range, 77.25-289.50) than the dissection side (median, 387.50; inter-quartile range, 278.25-544.50; $p<0.001$ ) (Table I and Figure 7). Pain scores were sometimes unobtainable in recovery as patients were too drowsy. However, even after excluding pain scores reported in recovery, overall pain scores remained lower for the guillotine side (area under the curve analysis, $n=85$; median, 200.50; inter-quartile range, 81.50-346.25) than for the dissection side (median, 388.50; inter-quartile range, 260.00-553.75; $p<0.001)$. Median pain scores at each time point were also lower on the guillotine side than the dissection side (Figure 7). Of those children who reported a difference in pain between sides 24 hours post-operatively $(n=63), 87$ per cent reported less pain on the guillotine side $(p<0.001$, binomial two-sided test).

\section{Complications and post-operative questionnaire}

There were no primary haemorrhages in either group.

There were three cases of secondary haemorrhage, all on the dissection tonsillectomy side; however, this difference did not reach statistical significance $(p>$ 0.1, McNemar two-sided test) (Table I). All secondary haemorrhages were managed conservatively with observation and intravenous antibiotics.

\begin{tabular}{|c|c|c|c|}
\hline & $\begin{array}{c}\text { TABLE I } \\
\text { STUDY FINDINGS }\end{array}$ & & \\
\hline Variable & Tonsil & chnique & $p^{*}$ \\
\hline & Guillotine & Dissection & \\
\hline Operative time & & & \\
\hline Procedure time (med (IQR); min) & $6.00(5.00-7.00)$ & $10.50(9.00-13.00)$ & $<0.001$ \\
\hline Intra-operative bleeding & & & \\
\hline Blood loss (med (IQR); mm) ${ }^{\dagger}$ & $1.00(0.00-2.00)$ & $17.00(10.00-24.75)$ & $<0.001$ \\
\hline Pts needing swabs \&/or diathermy (\%) & 63.0 & 100 & $<0.001^{\ddagger}$ \\
\hline Complications & & & \\
\hline Pts with $2^{\circ}$ haemorrhage (\%) & 0.00 & 3.00 & $>0.100^{\ddagger}$ \\
\hline Post-operative pain & & & \\
\hline Pain score (AUC; med (IQR))** & $172.00(77.25-289.50)$ & $387.50(278.25-544.50)$ & $<0.001$ \\
\hline Pts with less pain on $G$ than D side $(\%)^{\S}$ & 87.30 & 12.70 & $<0.001^{\#}$ \\
\hline
\end{tabular}

Medians (med) and inter-quartile ranges (IQR) are presented because data were non-parametric. ${ }^{*}$ Two-sided $p$ values (Wilcoxon test) unless otherwise specified, consistent findings also achieved using paired $t$-tests; ${ }^{\star} \mathrm{McNemar}$ test; ${ }^{\sharp}$ binomial test. ${ }^{*}$ Suction used in two cases but difference remained significant when these cases removed. ${ }^{* *}$ Area under the curve (AUC) used as summary measure of pain scores over time $(n=53) .{ }^{\S}$ Based on $n=63$, where difference in pain by side reported, at 24 hours post-operatively. Min $=$ minutes; Pts $=$ patients; $2^{\circ}=$ secondary; $\mathrm{G}=$ guillotine; $\mathrm{D}=$ dissection 


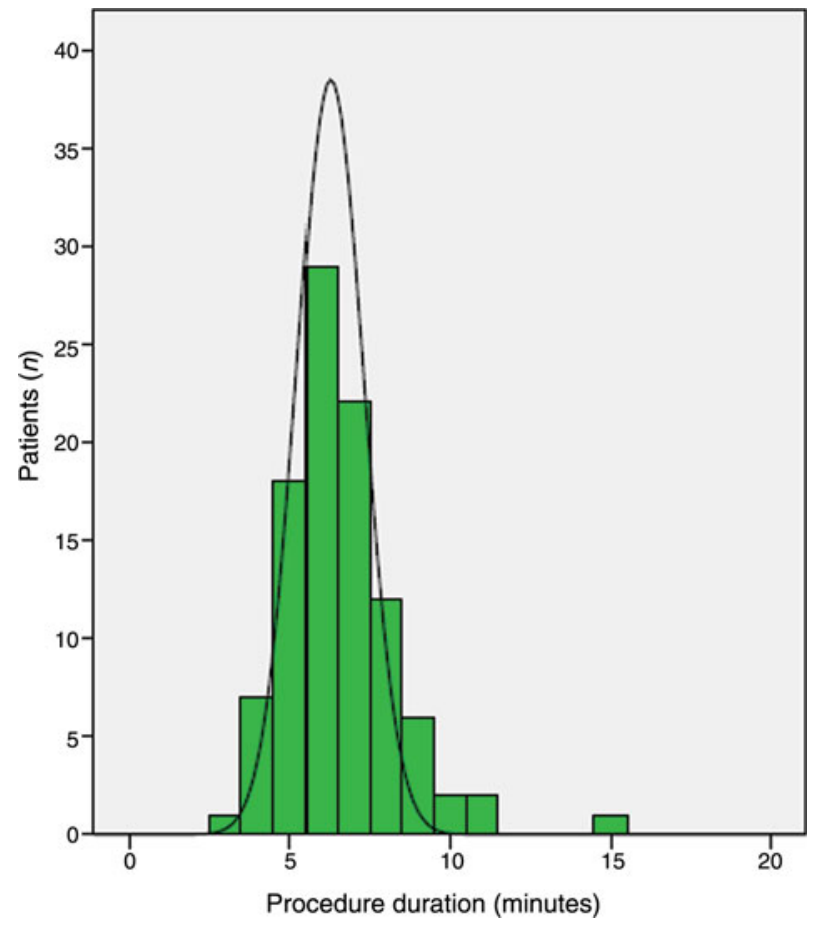

FIG. 3

Histogram of duration of guillotine procedure $(n=100)$.

\section{Discussion}

The aims of tonsillectomy are to remove the tonsils completely, with minimal haemorrhage, in a reasonable time, with as little post-operative pain and as few postoperative complications as possible.

Controversy remains over which is the optimum surgical tonsillectomy technique. Our study findings

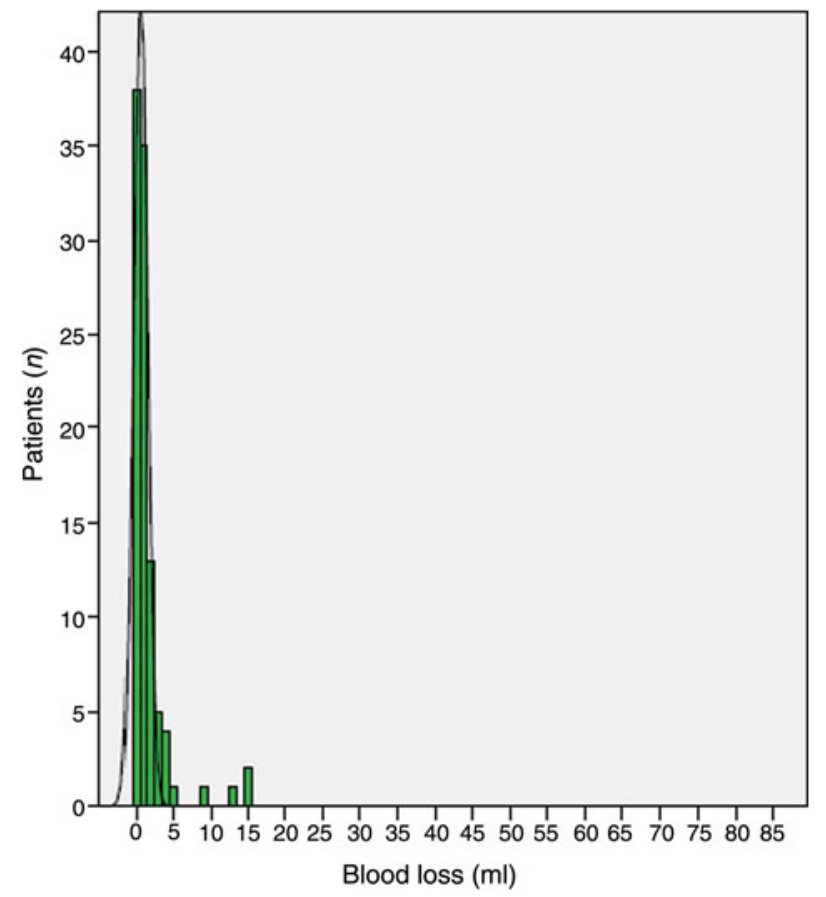

FIG. 5

Histogram of blood loss from guillotine procedure $(n=100)$.

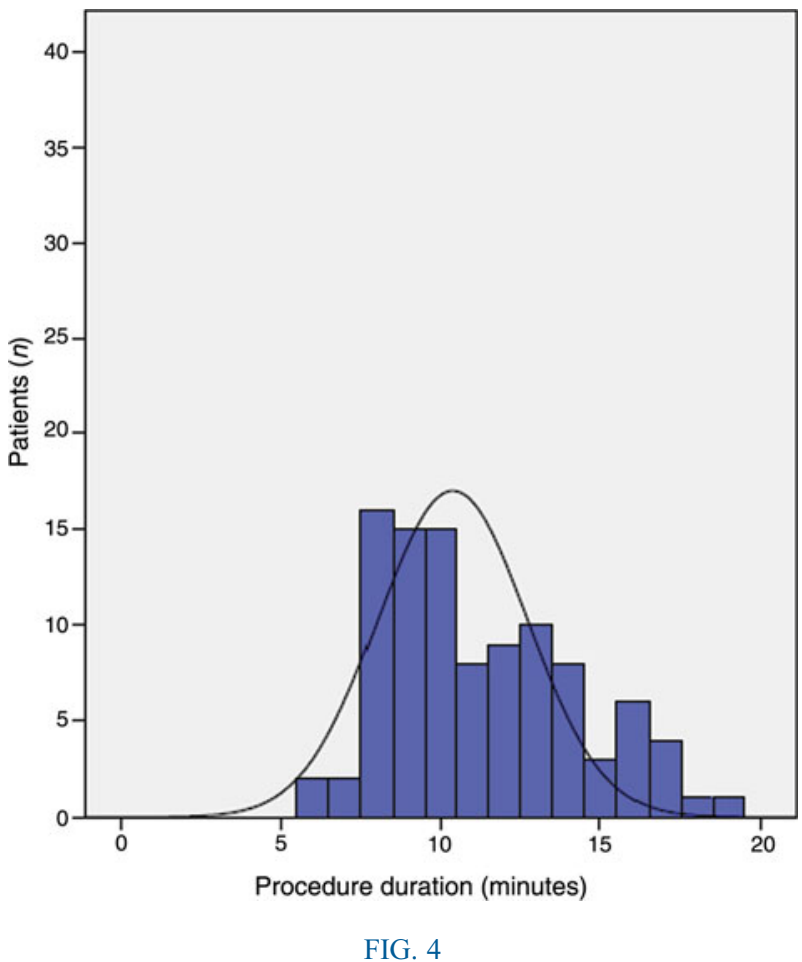

Histogram of duration of dissection procedure $(n=100)$.

indicate that use of the guillotine technique, in children with mobile tonsils, delivers a significant reduction in operative time, intra-operative blood loss and postoperative pain, compared with the dissection technique.

A previous study randomised children to have both tonsils removed by either the dissection or the

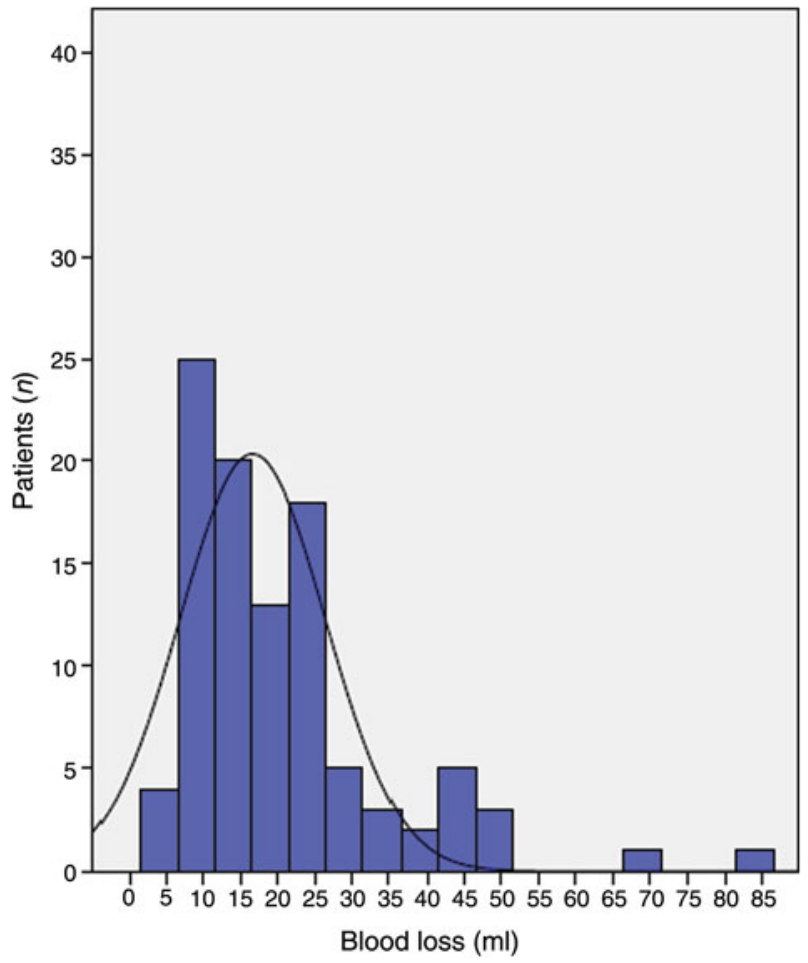

FIG. 6

Histogram of blood loss for dissection procedure $(n=100)$. 


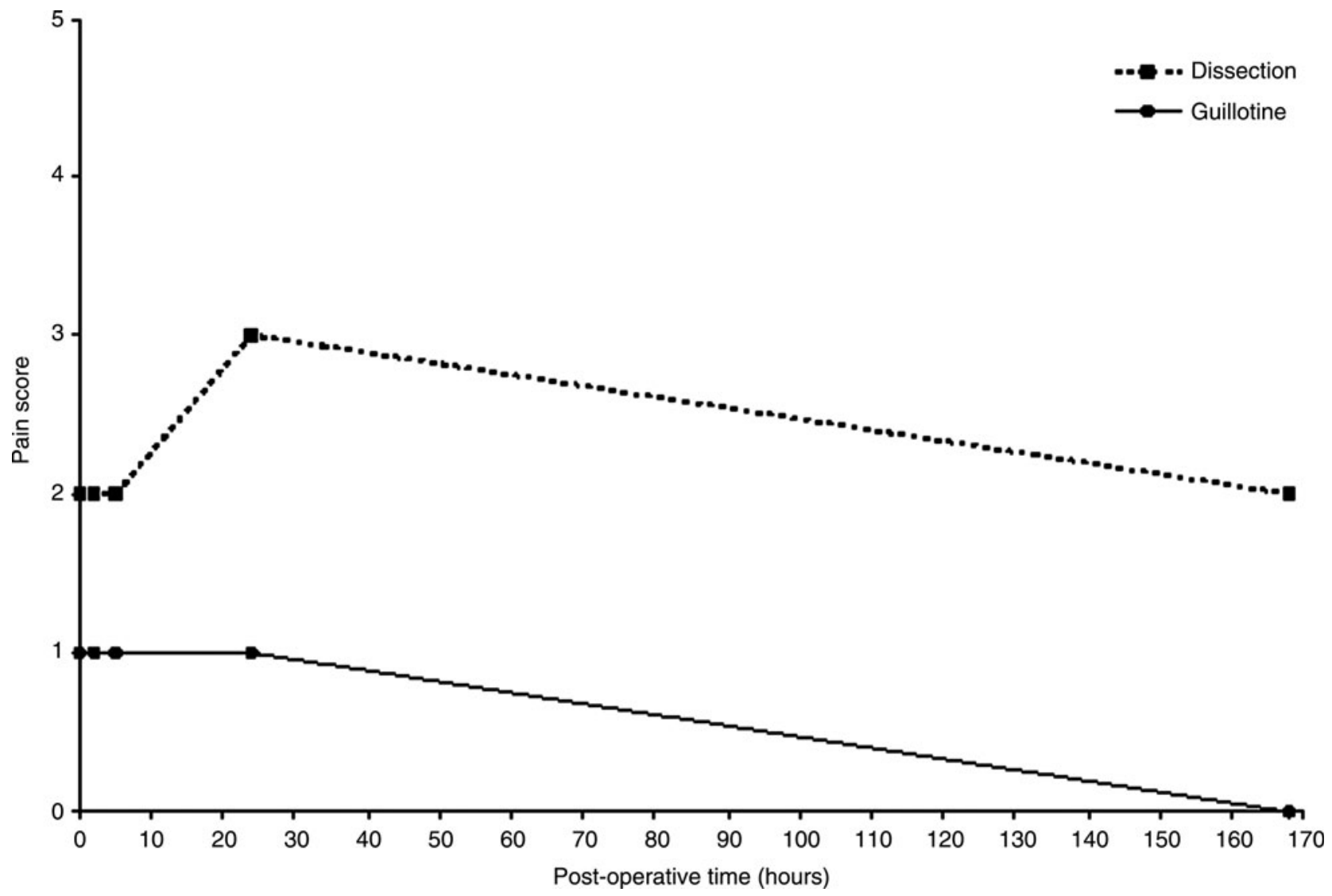

FIG. 7

Line graph of median post-operative pain scores, comparing guillotine and dissection techniques. Pain scores were obtained for 63 patients at 0 hours (i.e. in recovery), 93 at 2 hours, 91 at 5 hours, 98 at 24 hours (i.e. day one) and 99 at 168 hours (i.e. day seven).

guillotine technique, under general anaesthesia, and found that the guillotine technique was significantly quicker. ${ }^{6}$ These findings support those of the current study, which controlled for patient variability by using both techniques on the same patient.

In the current study, the second tonsil was not dissected until haemostasis was secured on the guillotine side. On the dissection side, more time was spent securing haemostasis, while on the guillotine side more time was spent observing to ensure that bleeding did not commence. Therefore, doubling the unilateral operative time to gain a figure for bilateral tonsillectomy operative time would potentially overestimate the operative time for both techniques. During bilateral dissection tonsillectomy, tonsil swabs can be placed in one tonsillar fossa after tonsil excision, to promote haemostasis, while the other tonsil is excised. More time, however, is likely to be saved during bilateral guillotine tonsillectomy, as both tonsils can be rapidly excised followed by a period of bilateral observation for delayed haemorrhage.

There is very little published data regarding intraoperative bleeding from guillotine tonsillectomy. In a recent, retrospective review of the results from Poole Hospital, there was minimal blood loss associated with bilateral guillotine tonsillectomy, with 54 per cent of patients requiring two or fewer tonsil swabs for haemostasis. ${ }^{4}$ Wake and Glossop randomised 50 children to undergo the dissection technique and 50 the guillotine technique, and found a statistically significant reduction in intra-operative blood loss with the latter. ${ }^{6}$ Weligodapola performed guillotine and dissection tonsillectomy on 50 patients younger than 20 years of age, with each patient undergoing both techniques such that one side acted as a control for the other. ${ }^{7}$ The author commented that intra-operative blood loss was less for the guillotine than the dissection technique, although this was a subjective observation and was not quantified. They noted that 50 ligatures were required on the collective dissection side versus 11 on the guillotine side. Unfortunately, no statistical analysis was performed to clarify the significance of these differences. Unlu et al. also reported less intra-operative bleeding for guillotine compared with dissection tonsillectomy. ${ }^{8}$ The haemostatic blade of Popper's guillotine may be responsible for the low intra-operative blood loss seen with the guillotine technique. In our study, the haemostatic blade was engaged and left in place for 2 minutes before the cutting blade was deployed. We believe this gave time for thrombosis within small vessels prior to deployment of the cutting blade, resulting in less bleeding when the tonsil was excised.

Post-operative pain is an important outcome when comparing tonsillectomy techniques. Standard dissection tonsillectomy involves essentially tearing the 


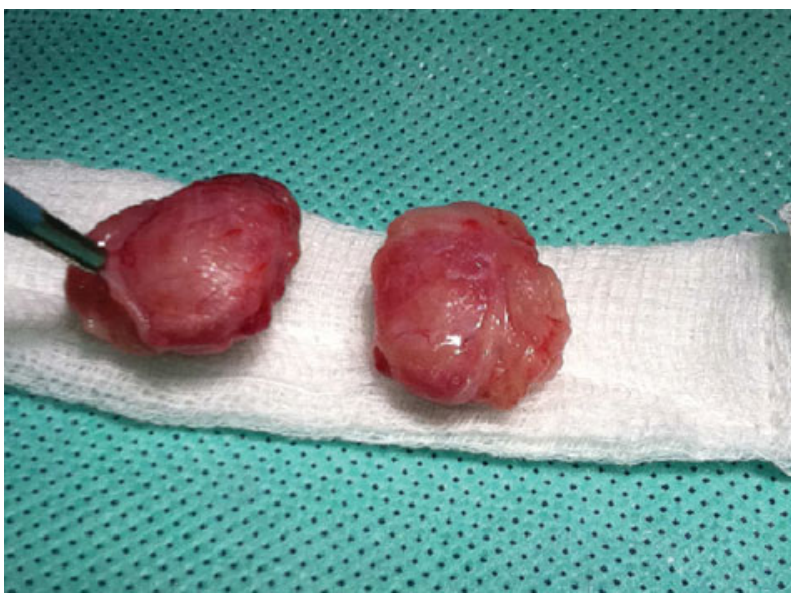

FIG. 8

Surgical photograph of a pair of tonsils removed using the guillotine (not from this study). The guillotine cuts through the loose connective tissue without breaking into the tonsil capsule and therefore, in trained hands, does not leave tonsillar remnants.

tonsil from its fossa. This inevitably results in the shearing of sensory nerve fibres and, consequently, marked post-operative pain. With Popper's guillotine, there is a single, sharp incision through loose areolar tissue, and where the tissue plane is well demarcated there is little damage to the underlying constrictor muscle (Figure 8). It is possible that this results in less nerve trauma and lower levels of post-operative pain. Alternatively, it is possible that the reduced amount of bipolar diathermy required in the guillotine group accounted for the reduction in pain. Our finding of reduced pain scores for guillotine tonsillectomy at all post-operative time points is consistent with that of Homer et al., who reported a low relative risk of 0.36 for moderately severe to severe pain within the first 24 hours, comparing guillotine to dissection tonsillectomy. ${ }^{9}$ Our findings are also supported by Wake and Glossop, who found reduced analgesia requirements in the guillotine group in the first two days post-tonsillectomy, and parental reports of reduced pain during recovery at home, compared with the dissection group. ${ }^{6}$ Weligodapola found that, in those patients who had one tonsil removed by guillotine and the other by dissection, over the first 48 hours the dissection side was reported to be the more painful by most patients. However, it was unclear as to the degree of blinding in this study and there was no statistical analysis of results. ${ }^{7}$

Secondary post-operative haemorrhage can be lifethreatening. Yuan et al. reported secondary haemorrhage in 1.16 per cent of 11140 guillotine tonsillectomies, compared with 1.47 per cent in those removed by the dissection technique. ${ }^{10}$ Other studies have reported conflicting findings. Carrick reported that guillotine tonsillectomy had no adverse effects on postoperative haemorrhage incidence, whilst Williams' review of 18184 tonsillectomies reported double the number of 'returns to theatre' for guillotine tonsillectomy compared with dissection tonsillectomy cases. ${ }^{11,12}$ Weligodapola, in a study of 50 patients, reported one secondary haemorrhage on the dissection side and none on the guillotine side. ${ }^{7}$ Wake and Glossop randomised 100 patients equally to guillotine or dissection methods, and observed no secondary haemorrhage with either technique. ${ }^{6}$ Our hospital is the only one with Otolaryngology services in our patient catchment area, and all our study patients were asked to return to our hospital if they experienced complications. Although the difference was non-significant ( $p>0.1, \mathrm{McNemar}$ two-sided test), we found a lower proportion of secondary haemorrhage for guillotine tonsillectomy ( 0 per cent) than dissection tonsillectomy ( 3 per cent), reflecting the earlier results of Roberts et al. ${ }^{13}$ The National Prospective Tonsillectomy Audit found that bipolar diathermy haemostasis carried a slightly increased relative risk of secondary haemorrhage compared with the use of ties alone. ${ }^{2}$ The difference in secondary haemorrhage rates may reflect a greater requirement for bipolar diathermy haemostasis in the dissection group. However, it must be reiterated that the difference between techniques in this respect did not achieve statistical significance.

- Guillotine tonsillectomy was widely used before the advent of modern anaesthesia

- Now, few surgeons are experienced in this technique

- This study found it reduces operative time, blood loss and post-operative pain, compared with dissection tonsillectomy

- Its advantages may justify more frequent usage

A previous study comparing guillotine and dissection techniques found no evidence of increased tonsillar remnants in the guillotine group. ${ }^{6}$ In our study, all cases were examined for remnants and palatal trauma intra-operatively, at the end of the procedure, and no such complications were found for either technique. We strongly suspect that historical reports of such complications following use of the guillotine resulted from rushed procedures prior to the use of airway protection, or from inadequate training of the operating surgeon.

\section{Conclusion}

Guillotine tonsillectomy was the predominant technique before the development of anaesthetic airway protection, and provided quick, relatively painless and relatively bloodless excision of the tonsils. In more recent times this technique has fallen out of favour, with claims from some individuals of excessive bleeding, palatal trauma and the production of tonsillar remnants (although these claims may largely be based on historical data gathered before the development of modern anaesthesia). Such claims are not supported 
by the results of our study and literature review, which indicate that modern-day guillotine tonsillectomy has favourable outcomes in many respects, compared with the dissection technique, with no evidence of increased side effects.

Given the findings of the recent National Prospective Tonsillectomy Audit, we have been forced to reexamine our use of the more recently introduced 'hot' techniques for tonsillectomy. Most have been found to be associated with elevated secondary haemorrhage rates, and as a result the trend has shifted towards use of cold steel techniques. Although the guillotine technique is not appropriate for scarred, fibrotic or immobile tonsils, the majority of paediatric tonsillectomy cases are likely to be suitable.

The present study demonstrates that, in well trained hands, the guillotine technique may offer a reduction in operating time, intra-operative haemorrhage and post-operative pain, compared with cold steel dissection. Not only does the guillotine technique benefit the individual patient, but the shorter operating time may also facilitate efficiency savings.

Given these advantages, the guillotine technique may have a justifiable place in the surgical repertoire for tonsillectomy, and we would encourage trainees to gain exposure to this elegant yet neglected technique.

\section{Acknowledgements}

We would like to thank Professor Peter Thomas for his statistical advice and support for this study. We would also like to thank members of the ENT and anaesthetic departments, and the admissions, theatre, ward and medical photography staff at Poole Hospital, who all made significant contributions to the study. We extend special gratitude to Mr V R Talati, who introduced Mr Jain to the haemostatic guillotine.

\section{References}

1 McGuire NG. A method of guillotine tonsillectomy with an historical review. J Laryngol Otol 1967;81:187-95
2 Lowe D, Van der Meulen J, Cromwell D, Lewsey J, Copley L, Browne $\mathrm{J}$ et al. Key messages from the National Prospective Tonsillectomy Audit. Laryngoscope 2007;117:717-24

3 Mathews J, Lancaster J, Sherman I, Sullivan GO. Guillotine tonsillectomy: a glimpse into its history and current status in the United Kingdom. J Laryngol Otol 2002;116:988-91

4 Sunkaraneni VS, Ismail-Koch H, Salib RJ, Jain PK. Guillotine tonsillectomy: a neglected technique. J Laryngol Otol 2009; 123:907-9

5 Jain PK. How we do it: Jain tonsil 'A' frame - a solution to slipping rods. Clin Otolaryngol 2005;30:274-5

6 Wake M, Glossop P. Guillotine and dissection tonsillectomy compared. J Laryngol Otol 1989;103:588-91

7 Weligodapola GS. Comparative study of tonsillectomy performed by guillotine and dissection methods. J Laryngol Otol 1983;97:605-6

8 Unlu Y, Tekalan SA, Cemiloglu R, Ketenci I, Kutluhan A. Guillotine and dissection tonsillectomy in children. J Laryngol Otol 1992;106:817-20

9 Homer JJ, Williams BT, Semple P, Swanepoel A, Knight LC. Tonsillectomy by guillotine is less painful than by dissection. Int J Pediatr Otorhinolaryngol 2000;52:25-9

10 Yuan CC, Yu DY, Jun TS, Quan CR. Guillotine tonsillectomy without anesthesia. Auris Nasus Larynx 1984;11:29-35

11 Carrick DG. Salicylates and post-tonsillectomy haemorrhage. J Laryngol Otol 1984;98:803-5

12 Williams RG. Haemorrhage following tonsillectomy and adenoidectomy. (A review of 18,184 operations). J Laryngol Otol 1967;81:805-8

13 Roberts C, Jayaramachandran S, Raine $\mathrm{CH}$. A prospective study of factors which may predispose to post-operative tonsillar fossa haemorrhage. Clin Otolaryngol Allied Sci 1992;17:13-17

Address for correspondence:

Dr Parmod K Jain,

Department of Otolaryngology,

Poole Hospital NHS Foundation Trust,

Longfleet Road,

Poole BH15 2JB, UK

Fax: $+44(0) 1202448410$

E-mail: parmod.jain@virgin.net

Mr P K Jain takes responsibility for the integrity of the content of the paper

Competing interests: The Jain Tonsil 'A' Frame and the Jain Insulated Tonsil Dissector and Pillar Retractor are inventions of Mr P K Jain and are manufactured by Avondale Surgical UK 